\title{
Advanced diagnostic techniques for thermal plasmas
}

\author{
J. R. Fincke \\ Idaho National Engineering Laboratory, Idaho Falls, Idaho 83415 USA
}

\begin{abstract}
Characterization of high power plasma devices and processes requires the direct measurement of heavy particle temperature, electron temperature, electron number density, plasma velocity, and composition. Traditional diagnostic techniques, such as emission spectroscopy, which rely on the assumption of local thermodynamic equilibrium are questionable at best for high velocity flow fields. Because of nonequilibrium and demixing effects, direct measurements which do not rely on assumptions of local thermodynamic equilibrium or composition are necessary.
\end{abstract}

\section{INTRODUCTION}

The dynamics of a flowing plasma, its chemical state, and interactions between the plasma and injected material are controlled by the major energy pools and the rate of energy transfer between them. The major energy pools for a $1 \mathrm{eV}$ atmospheric pressure thermal plasma are the kinetic energy of the electrons and heavy particles (neutrals and ions), and the energy which is required for the formation of ionized states and/or dissociation of molecular species. The electronically excited states account for only a small percentage of the total internal energy. The ions (and/or dissociated molecular species) are particularly important, since they constitute a significant fraction of the total energy, and due to finite recombination rates, are often not in equilibrium with kinetic temperatures in flowing plasmas ${ }^{1}$. Due to the relatively poor collisional coupling between the electrons and the kinetic energy of the heavy particles, the excess energy that the electrons may have or acquire in the recombination process can result in electron temperatures being elevated (by a few tenths of an $\mathrm{eV}$ at atmospheric pressure) over the kinetic temperature of the heavy species. At low electron densities the ground state is connected to the excited states through only the very high energy tail of the electron energy distribution. Hence only a few of the total number of electrons serve to mix the excited levels and the ions with the ground state. This can result in over population of the ground state. In addition, diffusion in the strong gradients in the periphery and transport of resonance radiation can also contribute to departures from equilibrium. The difficulties are compounded when the composition of the plasma is altered inhomogeneously by injection or entrainment ${ }^{2}$ and diffusion driven demixing ${ }^{3,4}$.

Traditional diagnostic techniques, such as emission spectroscopy, which rely on the assumption of local thermodynamic equilibrium are questionable at best for high velocity flow fields. To characterize modern devices and processes, techniques are required for the direct measurement of heavy particle temperature, electron temperature, electron number density, plasma velocity, and composition. In general, nonintrusive, laser based techniques are preferable. Because of nonequilibrium and demixing effects, direct measurements, which do not rely on assumptions of local thermodynamic equilibrium or plasma composition are necessary. In this paper some recent developments in the diagnostics of thermal plasma flow fields examined.

\section{RAYLEIGH AND COHERENT THOMSON SCATTERING}

High resolution lineshape analysis of elastically scattered laser light allows direct and nonintrusive measurement of the plasma velocity and gas or heavy particle temperature. ${ }^{5,6}$ In principle, the electron temperature and electron number density can also be determined. Interpretation of lineshape data does not depend on LTE assumptions or reliance on non-LTE models, although Maxwell-Boltzmann kinetic energy distributions are usually assumed for each species. Thus, scattering theory allows the electron and ion energy distributions to be characterized by different temperatures. The plasma velocity is obtained by choosing a scattering geometry such that the difference between the scattered light wave vector and the incident laser wave vector has a component along the flow velocity. The lineshape will then be Doppler shifted relative to the incident laser frequency and the bulk gas velocity is determined. 
The scattering of electromagnetic radiation by a medium is due to density fluctuations within the medium. ${ }^{7}$ In the case of ionized gases, density fluctuations of the atoms and ions give rise to Rayleigh scattering, while density fluctuations of the free electrons give rise to Thomson scattering. Density fluctuations of the free electrons have two components, one resulting from the thermal motion of the electrons themselves (electron feature), and the other due to electrostatic interactions with moving ions (ion or ion-acoustic feature). At high electron densities Thomson scattering from electrons is dominant, and at lower electron densities, Rayleigh scattering from the heavy species is dominant.

High resolution ion-feature lineshape measurements with a good signal-to-noise ratio (SNR), require the use of high peak power, narrow linewidth laser sources and high resolution analysis of the scattered light. A pulsed, doubled, injection seeded Nd:YAG laser with a nearly transform limited bandwidth of $\sim 100 \mathrm{MHZ}$ and a scanning, high finesse, Fabrey-Perot interferometer (F-PI) are ideal. A schematic of a typical experiment is shown in Fig. 1. A small amount of laser light is split off of the main beam and focused into the F-PI to act as a frequency reference from which Doppler shifts due to the gas velocity are measured.

A typical ion-feature lineshape measured in an atmospheric pressure argon plasma near the exit of a spray torch operating at $22 \mathrm{~kW}$ is shown in Fig. 2. The central narrow peak in the lineshape is the F-PI response to the reference laser beam. The curve represents the least squares fit of the data to the theory, which is a superposition of the Thomson scattering and Rayleigh scattering lineshapes. The symmetric double humped ion-acoustic feature is evident as is the Doppler shift from the laser frequency. Because of the insensitivity to electron temperature and number density the fit yields only ion temperature and bulk velocity.

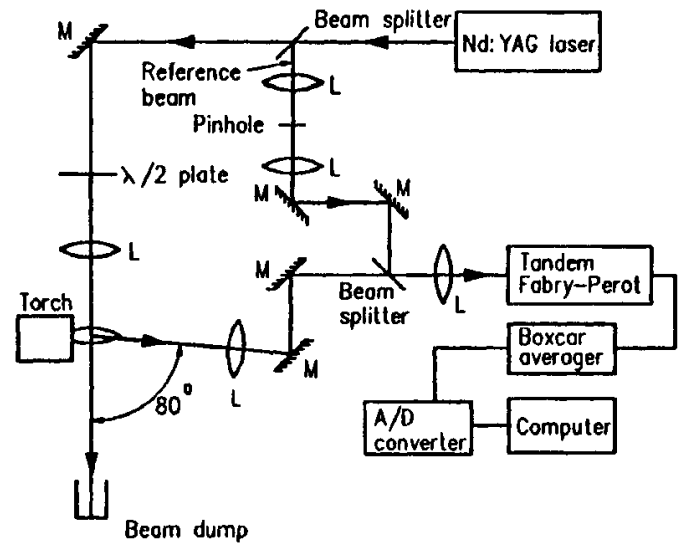

Fig. 1. Schematic of laser scattering experiment.

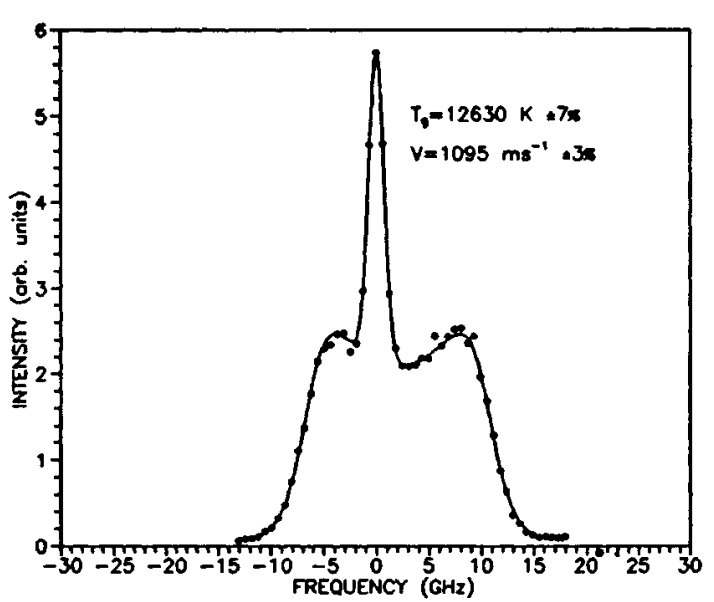

Fig. 2. Doppler shifted ion-acoustic feature for all argon, 900 A operating current.

The measurement of the electron feature requires much less resolution and a non-injection seeded Nd:YAG laser can be used in conjunction with a medium resolution grating spectrometer. Figure 3 contains a typical electron feature obtained using a $1.33 \mathrm{~m}$ spectrometer with a $600 \mathrm{l} / \mathrm{mm}$ grating and a gated-intensified twodimensional optical multichannel analyzer. The spectral resolution is $0.027 \mathrm{~nm}$ and the spectrum is averaged over 50 laser shots. The central, unresolved ion feature has been included to illustrate its relative magnitude. The least squares fit of the scattering theory to the electron feature yields electron number density and electron temperature. In measuring the electron feature the effect of laser heating on the electrons themselves must be accounted for. This is usually accomplished by measuring the electron feature at various laser powers and, since absorption of laser energy is a linear process (inverse bremsstrahlung), linearly extrapolating to zero laser power, Fig. 4.

The data of Figs. 2-4 all correspond to the same atmospheric pressure, argon plasma. At these conditions (pressure and electron density) the large apparent difference in heavy particle and electron temperature was unexpected. Kinetic modeling of the relaxation process ${ }^{1}$ using the classical formulation of electron-ion momentum transfer and the measured electron density indicates that the relaxation time from $T_{c}=2 e V$ and 


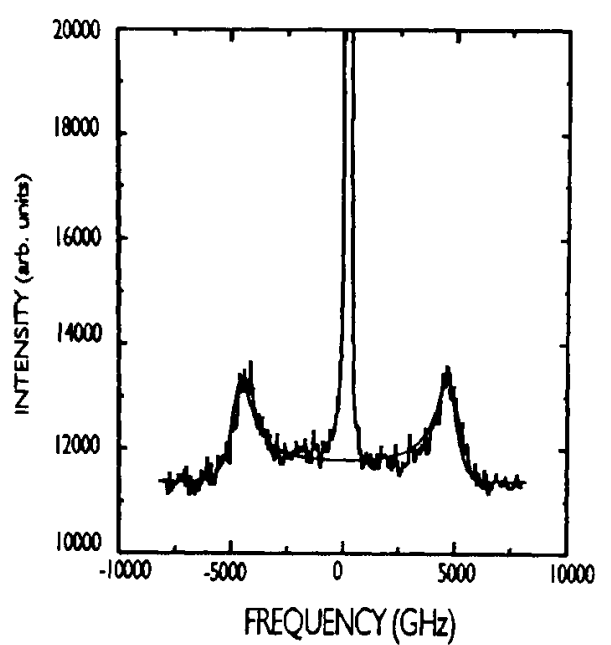

Fig. 3. Measured electron feature, $T_{e}=25,800 \mathrm{~K}$, $\mathrm{n}_{\mathrm{e}}=1.25 \times 10^{16} \mathrm{~cm}^{-3}$.

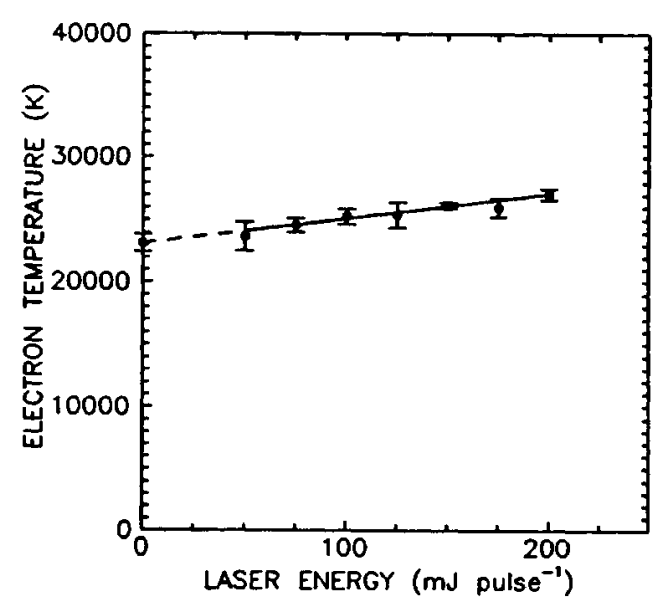

Fig. 4. Electron heating curve.

$T_{i}=1 \mathrm{eV}$ to equilibration is on the order of $100 \mathrm{~ns}$. At the measured plasma velocities $(1000 \mathrm{~m} / \mathrm{s})$ the distance traveled is approximately $0.1 \mathrm{~mm}$. At the calculated recombination rates the maximum difference, $T_{e}-T_{i}$, that can be supported by three body recombination $\left(\mathrm{Ar}^{+}+2 \mathrm{e}^{-}-\mathrm{Ar}+\mathrm{e}^{-}(+\mathrm{ke})\right)$ is only $0.1 \mathrm{ev}$. To sustain an electron/heavy particle temperature difference of $1 \mathrm{ev}$ by three body recombination the classic electron-ion momentum transfer rate must be decreased by a factor of at least 50 . The measured heavy particle temperatures are generally consistent with enthalpy probe and both single line, absolute intensity and Boltzmann plot emission spectroscopy results. The assumption of local thermodynamic equilibrium (LTE) which is required for interpretation of the emission data is reasonable at the nozzle exit. The electron number density obtained from the emission results assuming LTE, and Stark broadening measurements using the $\mathrm{H}_{\beta}$ line are also in reasonable agreement with each other $( \pm 10 \%)$ and are less than the laser scattering result by $50 \%$. The expected electron number density can also be calculated using the concept of partial local thermodynamic equilibrium (PLTE). ${ }^{8}$ In PLTE the higher energy excited states are in equilibrium with the electrons and the ion density, while the ground state may be under or over populated. Many, high density plasmas are known to be in approximately PLTE. ${ }^{8}$ Writing the excited state Saha equation and using the emission results for excited state population and the laser result for electron temperature indicates that the measured electron density (laser result) is lower than calculated by a factor of two. The laser measurement of electron temperature and density implies that this plasma should continue to ionize. This result is inconsistent with the fact that this is a recombining plasma. In addition, in the absence of a discharge current (post nozzle exit) the ionization process would rapidly cool the electrons to the ion temperature with an equilibration time on the order of $100 \mathrm{~ns}$ or less. These results indicate that the laser scattering measurements of electron temperature and density are inconsistent with accepted physics. The most likely areas to examine for an explanation of the observed inconsistency are the way in which the influence of laser heating is determined and in the scattering theory itself. Laser heating was examined by expanding the laser beam $10 \mathrm{X}$ for a $100 \mathrm{X}$ reduction in power density. Collecting the scattered signal from the entire beam cross section maintains acceptable signal levels at the expense of spatial resolution. This measurement, which should be essentially free of laser heating, yielded results consistent with the extrapolation in Fig. 4. The scattering theory, while widely accepted is, to the best of our knowledge, untested in the thermal plasma regime and its sensitivity to possible small deviations from MaxwellBoltzmann kinetic energy distributions and should be examined in detail. At this point the apparent inconsistency in electron and heavy particle temperature remains unresolved and is an active area of research in our laboratory.

\section{COHERENT ANTI-STOKES RAMAN SPECTROSCOPY}

Non-intrusive optical diagnostic techniques such as CARS have become standard tools in combustion research $^{9-11}$ and have been applied to thermal plasma jets ${ }^{1,12,13}$ as well as other plasma applications $s^{14,15}$. CARS has the advantage of high conversion efficiency, a coherent signal beam for high collection efficiency, excellent fluorescence and luminosity discrimination, and high spatial and temporal resolution. 
A CARS signal, Fig. 5, is generated when two laser beams at frequency $\omega_{1}$, (termed the pump beams) and one laser beam at frequency $\omega_{2}$ (termed the Stokes beam) interact through the third-order nonlinear susceptibility of the medium $X^{(3)}$ to generate an oscillating polarization and thus coherent radiation at frequency $\omega_{3}=2 \omega_{1}-\omega_{2}$. The intensity of the CARS signal, $I_{3}$, is proportional to $I_{3} \propto I_{1}^{2} I_{2}\left(3 X^{(3)}\right)^{2}$ where $I_{i}$ is the intensity at frequency $\omega_{r}$. The third-order nonlinear susceptibility, $X^{(3)}$ can be expressed as $X^{(3)}=X_{n}+X_{r}$, where $X_{n}$ is the nonresonant contribution and $X_{r}$ is the Raman resonant contribution. The nonresonant susceptibility is proportional to the number density of the species present, and is generally a slowly varying function of wavelength. The resonant contribution, far from electronic resonance, is given by $X_{\mathrm{r}}^{(3)}=\left(2 \mathrm{c}^{4} / \mathrm{h} \omega^{2}\right) \cdot \mathrm{N} \cdot \sum\left[\sigma \Delta(\mathrm{v}, \mathrm{j}) /\left(\omega_{\mathrm{r}}-\left(\omega_{1}-\omega_{2}\right)-\mathrm{i} \mathrm{G}_{\mathrm{v}, \mathrm{j}}\right)\right]$ where $\mathrm{N}$ is the number density of the species probed; $\Delta(\mathrm{v}, \mathrm{j})$ is the population difference between the states involved in the Raman Transition; $\sigma$ is the Raman scattering cross section; $\omega_{\mathrm{r}}$ is the frequency of a Raman active state; and $\mathrm{G}_{\mathrm{v}, \mathrm{j}}$ is the Raman half-width. When the frequency difference $\left(\omega_{1}-\omega_{2}\right)$ is close to the frequency of a Raman resonance of a species, $\omega_{n}$ the magnitude of the CARS radiation at $\omega_{3}$ is resonantly enhanced, resulting in a signature unique to that molecular species.

An injection seeded, frequency-doubled, Q-switched Nd:YAG is used both as a pump beam for the dye laser which generates the Stokes beam, and as the CARS pump beam. Rotational temperatures, which are expected to be in equilibrium with kinetic temperatures, are derived from the spectral distribution of the CARS signal and concentration is a function of signal strength. A rotationally resolved room temperature $\mathrm{O}_{2}$ CARS spectra is shown in Fig. 6. The CARS technique is particularly useful in entrainment studies and in quench regions where molecular species are forming.
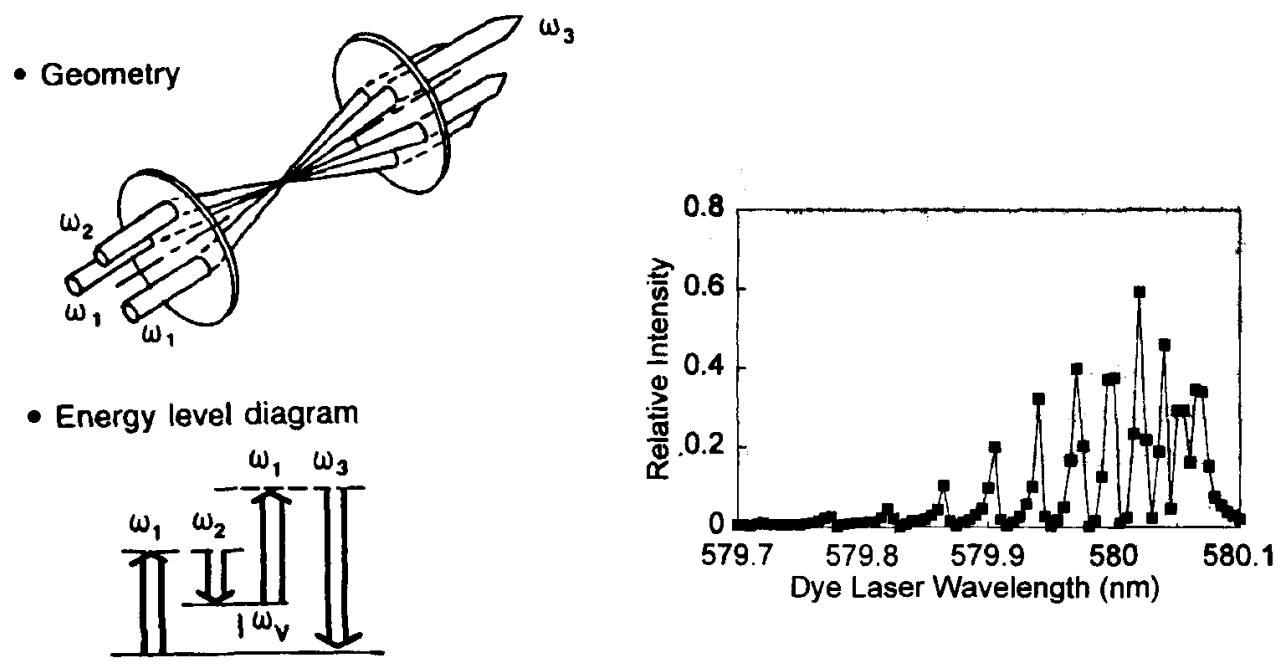

Fig. 5. Coherent anti-Stokes Raman spectroscopy.

Fig. 6. Rotationally resolved oxygen CARS spectra.

\section{LASER INDUCED FLUORESENCE}

Thermal plasmas used for materials processing are often composed of a mixture of an inert gas and a molecular gas such as hydrogen, oxygen, methane, or nitrogen, etc. When molecular species dissociate the gas enthalpy is increased, and the radicals produced play an important role in the chemistry of reactive plasmas. Their populations are, in general, not in equilibrium with the kinetic temperature of the plasma. Laser-induced fluoresence is useful for measuring concentrations of many atomic species. Laser-induced fluorescence (LIF) does not depend on LTE to determine species-concentration, however, LIF spectra can be quite complex if collisional redistribution of the population of the laser-excited state is significant, and photoionization of species in the plasma by the incident laser beam is a possible problem [16]. The measurement of concentrations is often further complicated by the fact that suitable excited states are often separated from the ground state by $8-10 \mathrm{eV}$ as is the case for atomic hydrogen and nitrogen.

Single-photon laser excitation of the ground state of atomic hydrogen is not possible with lasers presently available because the transitions are at vacuum-ultraviolet wavelengths. However, multiphoton excitation has been demonstrated in flames and plasmas [16,17]. The techniques are based on either single-wavelength 
2-photon, two wavelength $2+1$-photon, or single wavelength 3-photon excitation of the ground state to the $n=3$ or 4 level. The fluorescence transition monitored is either the $n=3$ to $n=2\left(H_{\alpha}\right)$ transition at 656.3 $\mathrm{nm}$ or the $\mathrm{n}=4$ to $\mathrm{n}=2\left(\mathrm{H}_{\beta}\right)$ transition at $486.1 \mathrm{~nm}$. The experimental setup for 2-photon excitation is considerably simpler than for $2+1$-photon excitation. Hence, the two $205 \mathrm{~nm}$ excitation scheme, Fig. 7, is preferred. The $205 \mathrm{~nm}$ laser wavelength is generated by frequency-tripling the $615 \mathrm{~nm}$ output of a $\mathrm{Nd}$ :YAG pumped pulsed-dye laser. Frequency tripling was accomplished using a $\mathrm{KD}{ }^{*} \mathrm{P}$ crystal to frequency double the fundamental dye laser output followed by mixing with the fundamental in a BBO crystal. Approximately $0.9 \mathrm{~mJ}$ pulse $\mathrm{e}^{-1}$ of laser energy was obtainable at $205 \mathrm{~nm}$ using a pump energy of $300 \mathrm{~mJ}$ pulse $\mathrm{s}^{-1}$ at $532 \mathrm{~nm}^{18}$. A schematic of the experimental set up is given in Fig. 8 .

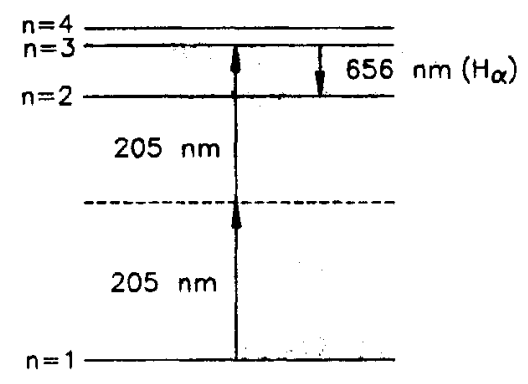

Fig. 7. Energy level schematic for twophoton excitation of atomic hydrogen.

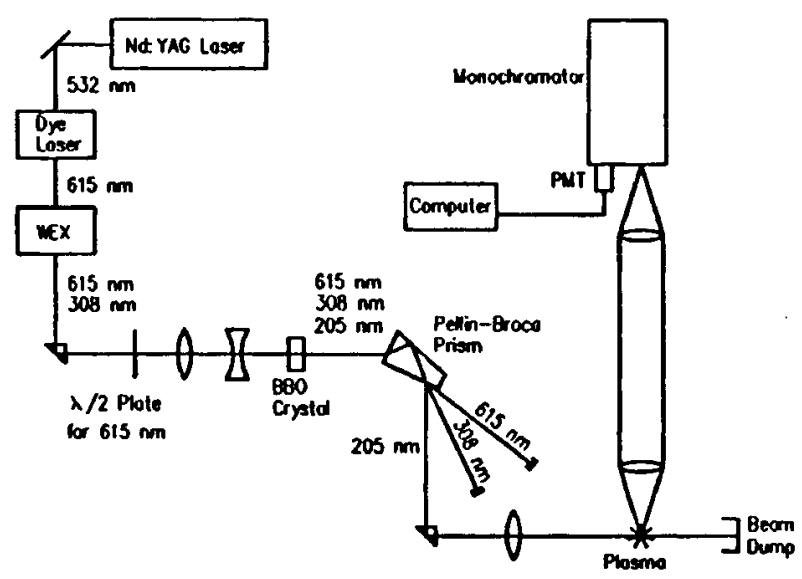

Fig. 8. Schematic of two-photon fluorescence experiment.

The dependence of the 2-photon fluorescence signal on laser intensity is quadratic if the signal is far from saturation and photoionization is not significant [16]. Figure 9 shows the 2-photon LIF signal strength at the $656 \mathrm{~nm}$ line of atomic hydrogen in a free-burning atmospheric argon-hydrogen arc operating over a water-cooled copper anode as a function of laser energy ${ }^{18}$. At low laser energies, the relationship between the signal and laser energy is quadratic. At higher laser energies, the signal begins to saturate.

Figure 10 contains the measured radial profiles of the atomic hydrogen density at $4 \mathrm{~mm}$ below the cathode of a free-burning arc for $1 \%$ and $5 \%$ hydrogen by mole fraction in argon ${ }^{18}$. The apparent hydrogen density decreases drastically near the arc center. The measurement is compromised by Stark broadening of the $\mathrm{H}_{\alpha}$ fluorescence signal in this region. The electron density in atmospheric-pressure free-burning arc discharges is on the order of $1.5 \times 10^{16} \mathrm{~cm}^{-3}$ in the arc column. At these electron densities, the Stark-broadened linewidth of the $H_{\alpha}$ transition is about $1 \mathrm{~nm}$, which is much broader than the bandwidth of the monochromator hence, only a small portion of the of Stark broadening signal overlaps with the bandwidth of the monochromator. The electron density decreases rapidly with increasing radial position, and hence

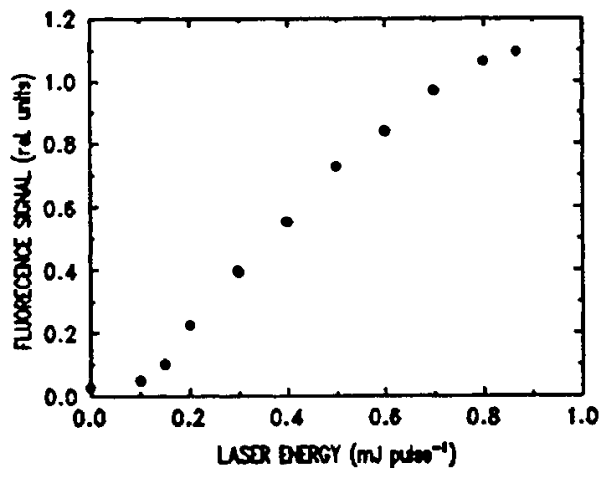

Fig. 9. Two-photon LIF signal as a function of laser energy.

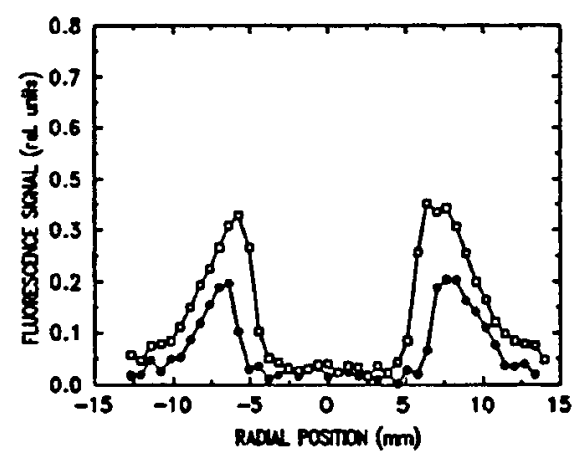

Fig. 10. Relative atomic hydrogen density, open squares and solid circles are for $5 \%$ and $1 \%$ hydrogen in argon, respectively. 
also the degree of Stark broadening of the $\mathrm{H}_{\alpha}$ transition. It was verified that the linewidth of the peak fluorescence signal was less than $0.05 \mathrm{~nm}$ at radial positions greater than $5 \mathrm{~mm}$. Furthermore, only the $\mathrm{H}_{\alpha}$ transition was observed indicating collisional redistribution of the population of the laser-excited state was not significant at these locations.

\section{SUMMARY}

Plasma flow field characterization requires measurements of velocity, composition, and independent measurement of electron and heavy particle temperature. Many of the laser based techniques that are used in combustion research are applicable but, in general, some care must be exercised. For instance, LIF measurements of light atom concentrations can be compromised by Stark broadening and the direct laser scattering measurement of electron temperature and concentration exhibits an apparent inconsistency with heavy particle temperatures which remains unresolved at this time.

\section{ACKNOWLEDGMENT}

This work was supported by the U. S. Department of Energy, Office of Energy Research, Office of Basic Energy Sciences, Division of Engineering and Geosciences, under DOE Idaho Operations Office, Contract No. DE-AC07-94ID13223.

\section{REFERENCES}

1. T. E. Repetti, J. R. Fincke, and W. E. Neuman, Heat Transfer in Thermal Plasma Processing, HTD-Vol. 161, K. Etemadi and J. Mostaghimi, Eds., ASME, 167 (1991).

2. E. Pfender, J. R. Fincke, and R. Spores, Plasma Chem. and Plasma Proc., 11, 529 (1991).

3. J. R. Fincke, W. D. Swank., and D. C. Haggard., J. Thermal Spray Tech. .

4. J. R. Fincke, C. H. Chang, W. Swank, and D. Haggard, Int. J. Heat Mass Transfer. 37, 1673 (1994).

5. S. C. Snyder, L. Reynolds, G. Lassahn, J. Fincke, C. Shaw, and R. Kearney, R., Phys. Rev. E, 471996 (1993).

6. S. C. Snyder, L. D. Reynolds, J. R. Fincke, G. D. Lassahn, J. D. Grandy, and T. E. Repetti, Phys. Rev. E $\underline{50}, 519$ (1994).

7. H. J. Kunze, "The Laser as a Tool for Plasma Diagnostics," in Plasma Diagnostics, edited by W. LochteHoltgreven, American Elsevier Publishing Co., Inc., New York, (1968).

8. C. H. Kruger, T. Owano, and M. Gordon, Pure and Applied Chemistry, 62, 1833 (1990).

9. M. D. Levenson, Introduction to Nonlinear Laser Spectroscopy, Academic Press, (1982).

10. R. R. Antcliff, and O. Jarrett, Rev. Sci. Instr. 58, 2075 (1987).

11. A. C. Eckbreth, and T. J. Anderson, Applied Optics, 24, 2731 (1985).

12. J. R. Fincke, R. Rodriguez, and C. G. Pentecost, Plas. Proc. and Syn. Mat. III, Materials Research Society Symposia Proceedings Vol. 190, Materials Research Society, 184 (1991).

13. J. R. Fincke and C. G. Pentecost, Heat Transfer in Thermal Plasma Processing, HTD-Vol. 161, K. Etemadi and J. Mostaghimi, Eds., ASME, 101 (1991).

14. S. O. Hay, et al, ISPC-10, 1.2-4 p.1 (1991).

15. Y. Kornas, et al, Plasma Chem. and Plasma Proc., 11, 171 (1991).

16. J. E. M. Goldsmith and N. M. Laurendeau, Opt. Lett. $\underline{15}$, 576 (1990).

17. D. Wagner, V. Schulz-von der Gathen, H. F. Döbele, and A. T. Young, ESCAMPIG 92: Eleventh European Sectional Conference on the Atomic and Molecular Physics of Ionized Gases, St Petersburg, Russia, 1992, edited by L. Tsendin (European Physical Society, Geneva), p.273.

18. S. C. Snyder, et al, Phys. Rev. E submitted. 